\title{
TEOAE in Disorders of Ear Conduction Apparatus at Non-compensated and Compensated Pressure in Cavum Tympani \\ A Karić ${ }^{1}, \mathrm{~S}$ Đoković ${ }^{2}, \mathrm{~N}$ Dimić ${ }^{3}, \mathrm{R}$ Savic $^{3}$
}

\begin{abstract}
Objective: Transitory otoacoustic emissions (TEOAE) can be significant for diagnosing the hearing defect and for monitoring healing treatment for some diseases of ear conduction apparatus. They were investigated for non-compensated and compensated pressure in a middle ear in the two groups of diseases (inflammatory and non-inflammatory).

Methods: Following TEOAE parameters were analysed: amplitude (A), correlational response (CR), stimulus level (STL), relationship between stimulus and noise $(\mathrm{S} / \mathrm{N})$ and level of pressure. Relationship between stimulus and noise in $\mathrm{dB}$ response at different frequencies $(0.5,1.5,2.5$ and $3.5 \mathrm{kHz})$ was observed.
\end{abstract}

Results: Pressure compensation caused higher values of all TEOAE parameters in relation to the parameters of emission at ambiental pressure. Pressure linear regression effect on parameter values indicates linear decrease in the value of amplitude (A) and relationship of stimulus and noise at frequency of $1.5 \mathrm{kHz}$ for $0.02 \mathrm{~dB}$ SPL and $0.03 \mathrm{~dB}$ when the pressure was increased by one daPa. Within the groups of diseases, registered emission of $\mathrm{CR}$ and STL at pressure compensation was higher $(\mathrm{p}<0.01)$ in both groups. Pressure compensation caused a statistically significantly higher value of the relationship of stimulus and noise $(\mathrm{S} / \mathrm{N})$ only in the group of non-inflammatory disorders $(\mathrm{p}=0.0312)$. Within middle ear inflammatory disorders difference has not been found only between the relationship of stimulus and noise at frequency of $0.5 \mathrm{kHz}$. Compensational treatment in non-inflammatory diseases caused significantly higher relationship of stimulus and noise at all investigated frequencies. 
Conclusion: Pressure compensation in middle ear had a significant effect on obtaining TEOAE responses and their size.

Key words: Inflammatory and non-inflammatory disease, middle ear, otoacoustic emissions, pressure compensation

From: ${ }^{1}$ Health Center "New Belgrade", Goce Delčeva 30, 11000 Belgrade, Serbia, ${ }^{2}$ University of Belgrade, Faculty of Special Education and Rehabilitation, Visokog Stevana 2, 11000 Belgrade, Serbia, ${ }^{3}$ University of Belgrade, Faculty of Agriculture, Nemanjina 6, 11000 Belgrade, Serbia.

Correspondence: Dr R Savić, Faculty of Agriculture, University of Belgrade, Nemanjina 6, 11000 Belgrade, Serbia. E-mail: savic@agrif.bg.ac.rs

\section{INTRODUCTION}

Otoacoustic emissions (OAE) are widely applied today and have become an essential segment of complete auditory processing. Transient otoacoustic emission (TEOAE) is a segment of the class of evoked otoacoustic emissions and is generated as a response of cochlear to brief duration incentive signal in the external auditory canal (1) and like the other otoacoustic emissions can show the state of sensory segment of auditory apparatus. Their frequencies are not specific what is in contrast to DPOAE (distortion products).

Comparative analysis of (2) which included 579 newborn babies exposed to TEOAE and DPOAE screening showed a significantly strong positive relationship between these two procedures. By the application of the Spearman Rank's correlation coefficient they determined the pass index of $95 \%$ for TEOAE and of $91 \%$ for DPOAE. Comparing these two procedures showed that $91 \%$ of newborn babies passed both procedures, $4.5 \%$ babies passed only TEOAE and $0.5 \%$ babies passed only DPOAE, while $4 \%$ passed neither procedure.

We use them when we wish to test an entire cochlear since their incentive signal is a brief duration impulse signal which can prompt a whole basilar membrane. They represent 
complex acoustic incidents which can be registered in almost every human individual with normal hearing. The energy in the region of frequencies close to $1500 \mathrm{~Hz}$ dominates the response to acoustic click stimulus and amplitude of response is in relationship to magnitude of stimulus in a complex relationship (1).

Otoacoustic emissions are widely used in early diagnostics of hearing defects. Their application is non-invasive, of brief duration, can be done during sleep what is of special benefit to newborn babies and little children. Correct and early diagnosis of hearing defects is a precondition for starting timely habilitation of hearing and consequent correct morphofunctional development of complete auditory system, then of speaking and intelectual and emotional development.

When pathology of a middle ear is in question OAE are used to monitor recovery of acute or secretory chronic otitis media. In a disease evolution tone audiometry is being first recovered, then tympanometry and in the end otoacoustic emissions, therefore their normalisation can be considered as a sign of final healing (3).

The research conducted by a group of authors has shown that conduction pathology has a greatest influence on transfer characteristic in the range of medium frequencies therefore its most prominent influence on TEOAE is in the range of medium frequencies (4). Emission is most prominent in the range of medium frequencies meaning that it is immediately dependent on the function of middle ear which takes part in transfer of recurrent transmission of emission response of cochlear to stimulus (5).

There are various middle ear defects: perforation of tympanic membrane, negative pressure in middle ear, otitis media acute, chronic suppurative and non-suppurative otitis media, chronic secretory otitis media, catarrh or auditory tube, fixation - discontinuity of the chain of auditory ossicles, tumours, otosclerosis, tympanosclerosis, injure of mastoid region, etc. The application of otoacoustic emissions in differential diagnostics of sensorineural 
deafness is primarily in differentiating cochlear and retrocochlear defects. Some research (6) suggest that the method of transitory otoacoustic emisions can be reliable in detection and sensorineural and conductive hearing loss. Otoacoustic emissions can be registered in proper function of middle and inner ear, while on the contrary, they are absent or reduced in defects of cochlear and/or middle ear, even in less severe forms.

The aim of this study was to determine the value of TEOAE parameters at noncompensated (ambiental) and compensated pressure. In addition, the differences in registered parameters of otoacoustic emissions within diseases of ear conduction apparatus were discussed.

\section{MATERIAL AND METHODS}

The research was conducted at the Faculty of Medicine of Belgrade, Clinic of Otorhinolaryngology and Maxillofacial Surgery, Department of Audiology and Vestibulology of the Clinical Centre of Serbia, in the period from 2004-2010. The research included 150 patients (79 males and 71 females). Patients average age was 27.95 years with variation interval from 2 to 71 years. The study conducted in accordance with the local Ethics Committee. For procedure used in this study, consent was obtained from the patients according with the ethical guidelines.

In audiological diagnostics, besides anamnesis and standard clinical otorhinolaryngological (ORL) examination, tympanometry (Madsen Zodiak 201) was also applied in order to confirm or not confirm the defects at the level of middle ear or disfunction of Eustachian tube and, if necessary, a liminar tonal audiometry (Madsen Orbiter 922).

Measuring of TEOAE parameters was performed by means of Hortmann device. Following TEOAE parameters were analysed: amplitude (A; dB SPL), correlation response 
(CR; \%), level of stimulus (STL; dB SPL), relationship of stimulus and noise $(\mathrm{S} / \mathrm{N} ; \mathrm{dB})$ and level of pressure. The relationship of stimulus and noise in $\mathrm{dB}$ response to frequencies of 0.5 , $1.5,2.5$ and $3.5 \mathrm{kHz}$ was shown as well.

The size of TEOAE parameters was observed at non-compensated (ambiental) and compensated pressure in cavum tympani. All persons tested were divided into the two groups of diseases of conduction apparatus: one group with inflammatory $(Z)$ and the other group with non-inflammatory diseases (NZ). The ratio of the number of persons with inflammatory and with non-inflammatory diseases was 97:53. Inflammatory diseases included: otitis media acuta (OMA), otitis media secretoria (SOM), otitis media chronica non-suppurativa (HOMn), otitis media chronica suppurativa (HOMa), dysphunctio tubae auditivae (DT). The group of non-inflammatory diseases included: interruption, i.e. discontinuity of the chain of auditory ossicles (DL), trauma of mastoid region (DAM), tumours of external and middle ear (TUM), otosclerosis (OS) and tympanosclerosis (TS).

Data were processed in SAS 9.3 statistical package (7). Basic statistical parameters were calculated (mean value, standard deviation and variation interval). Testing of arrangement of frequencies was performed by use of non-parametric $\chi^{2}$ test. Effect of groups of diseases analysed using parametric ANOVA test. The relationship of measured TEOAE parameters was done by a Pearson correlation coefficient. Pressure effect on the values of otoacoustic emissions was observed as a linear regression effect. Comparison of mean values of registered TEOAE parameters was done by t-test. 


\section{RESULTS}

Within all the persons tested between the two groups of diseases of conduction apparatus: inflammatory (Z; 65\%) and non-inflammatory (NZ; 35\%) a statistically significant difference in frequencies of manifesting disorders $\left(\chi^{2}=12.907, p<0.0001\right)$ was determined.

Within each group of disorders, different changes of ear conduction apparatus (Figure 1) were diagnosed during test. Comparing the frequencies of diagnosed disorders, a statistically significant differences were determined between individual diseases $\left(\chi^{2}=27.867\right.$; $\mathrm{p}=0.001)$.

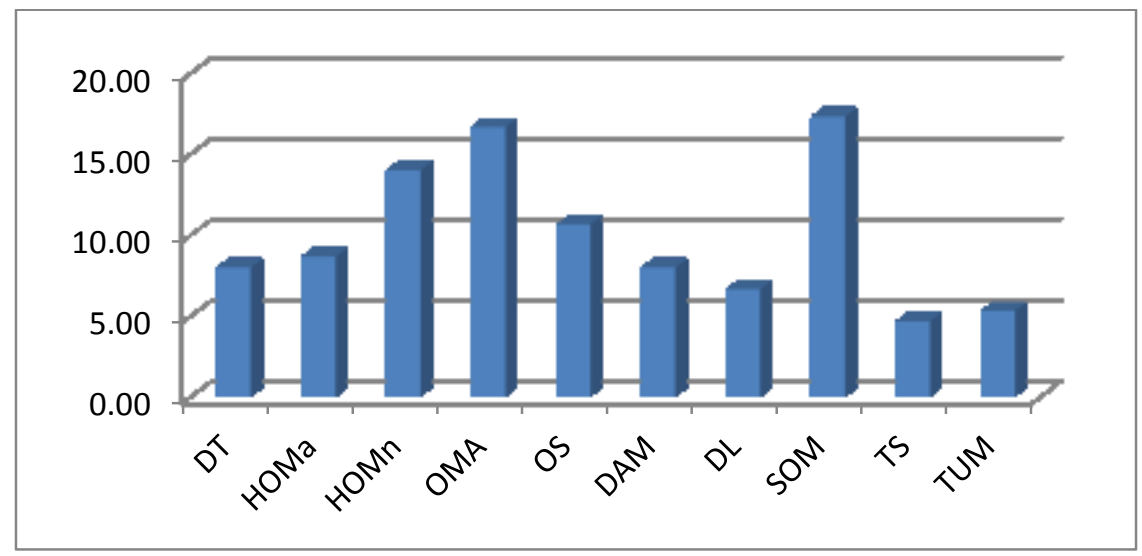

Figure 1. Distribution of frequency of disturbances of conductive apparatus according to the established diagnoses

Distribution of persons by male and female sex within groups ( $\mathrm{Z}$ and NZ) of disorders of conduction apparatus of ear indicates that there are no statistically significant differences between the two analysed groups $\left(\chi^{2}=1.955, \mathrm{p}=0.1620\right.$; Figure 2$)$.

Mean values and variability of measured TEOAE parameters indicate differences, depending whether the measuring was performed with or without pressure compensation (Table 1). Pressure compensation caused higher values of all TEOAE parameters in relation to emission parameters at atmospheric pressure. Linear regression of the effect of pressure on the values of parameters indicate linear decrease of values of amplitude (A) and relationship 
of stimulus and noise at the frequency of $1.5 \mathrm{kHz}$ for $0.02 \mathrm{~dB}$ SPL and $0.03 \mathrm{~dB}$, at increasing pressure by one unit $(1 \mathrm{daPa})$.

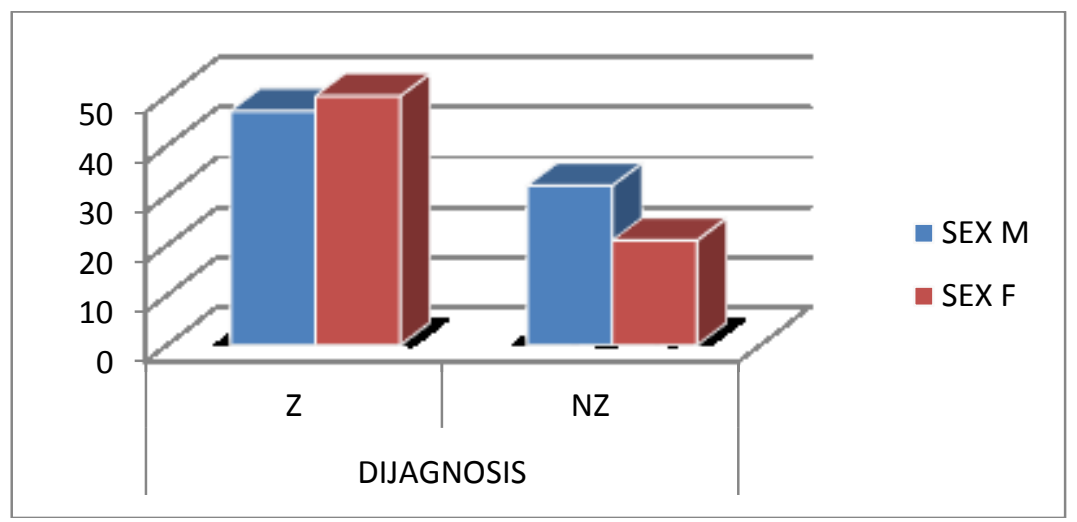

Figure 2. The frequency of respondents by sex and by groups of diseases (inflammatory- $Z$ and non-inflammatory-NZ)

Between measured TEOAE parameters, regardless pressure compensation there existed a correlation of different strength (Table 2).

Table 1. The average value and variability of parameters TEOAE at non-compensated and compensated pressure

\begin{tabular}{lccccccc}
\hline \multirow{2}{*}{ Parameters } & \multicolumn{3}{c}{ TEOAE at compensation } & \multicolumn{3}{c}{ TEAOE at non-compensation } \\
\cline { 2 - 8 } & $\overline{\mathbf{x}}$ & SD & Interval & bPREss & $\overline{\mathbf{x}}$ & SD & Interval \\
\hline Pressure (daPa) & -129.24 & 120.41 & $-361.00-195.00$ & - & - & - & - \\
CR (\%) & 44.99 & 39.62 & $-63.00-99.00$ & $0.03^{\text {ns }}$ & 24.87 & 37.30 & $-79.00-98.00$ \\
STL (dB SPL) & 64.84 & 23.96 & $-52.00-89.10$ & $0.00^{\text {ns }}$ & 49.87 & 34.37 & $-42.00-82.00$ \\
A (dB SPL) & 15.29 & 13.34 & $-16.00-90.00$ & $-0.02^{*}$ & 11.88 & 12.34 & $-23.00-66.00$ \\
S/N (dB) & 6.78 & 11.37 & $-19.00-81.00$ & $-0.01^{\text {ns }}$ & 4.81 & 7.60 & $-11.00-61.00$ \\
S/N_0.5 (dB) & 2.17 & 6.59 & $-11.00-21.00$ & $-0,00^{\text {ns }}$ & 0.25 & 6.37 & $-27.00-18.00$ \\
S/N_1.5 (dB) & 6.89 & 12.52 & $-11.00-78.00$ & $-0,03^{* *}$ & 0.87 & 7.40 & $-21.00-22.00$ \\
S/N_2.5 (dB) & 4.59 & 9.29 & $-48.00-31.00$ & $-0,01^{\text {ns }}$ & 1.40 & 7.56 & $-20.00-19.00$ \\
S/N_3.5 (dB) & 4.31 & 7.88 & $-17.00-27.00$ & $-0,00^{\text {ns }}$ & 1.17 & 7.54 & $-21.00-21.00$ \\
\hline CR-Co
\end{tabular}

CR- Correlation Response, STL- Stimulus Level, A- Amplitude, S/N- Proportion of Stimulus and Noise, S/N ( $0.5, \ldots 1.5, \ldots 2.5,3.5 \mathrm{kHz})$ - Proportion of Stimulus and Noise by different frequency, $\overline{\mathrm{x}}-$ Mean, SD- Standard Deviation, $\mathrm{p}_{\text {ress }}$ - Linear regression effect of pressure on TEOAE parameters value; Statistical significance: ns- not significant, ${ }_{\text {- }} \mathrm{p}<0.05,{ }^{*}$ - $\mathrm{p}<0.01$. 
By comparing the groups of diseases $(\mathrm{Z}$ and $\mathrm{NZ})$ within both treatments with pressure (non-compensated and compensated) a statistically significant differences $(p>0.05)$ in registered TEOAE parameters were not determined. Mean value of otoacoustic CR emissions was significantly higher with pressure compensation, regardless the disorder of ear conduction apparatus (Figure 3). Within diseases a registered emission at compensation was higher by $18.06 \%(\mathrm{p}=0.0001)$ i.e. $23.85 \%(\mathrm{p}<0.0001)$ in inflammatory, i.e. noninflammatory processes in middle ear. Statistically significant differences $(p<0.0001$ and $\mathrm{p}=0.0013$ ) existed also in STL, where value of this parameter at pressure compensation was higher in both groups of diseases.

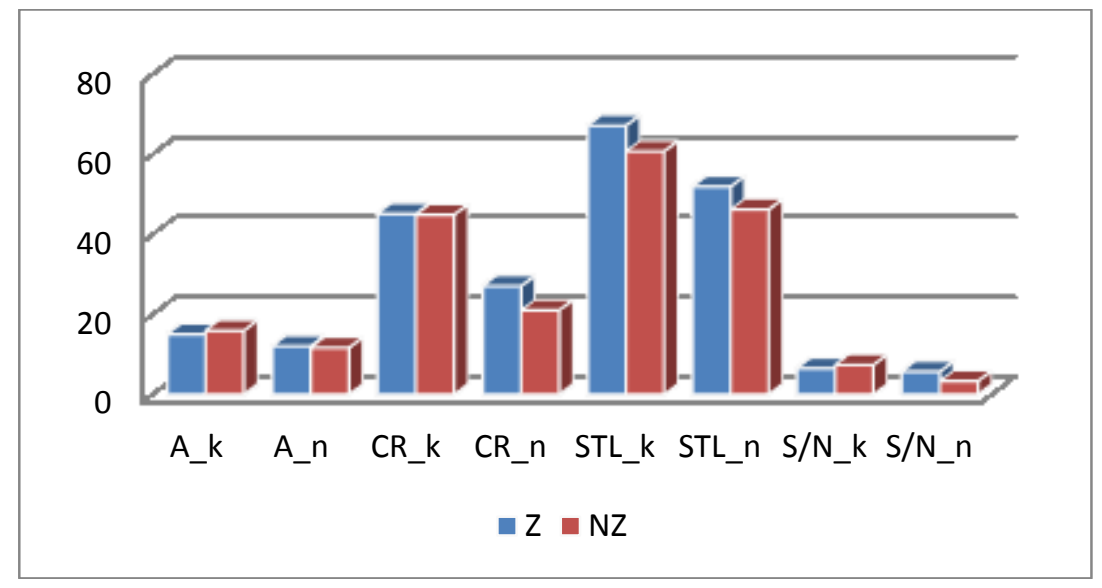

Figure 3. The average value of TEOAE: Amplitude-A (dB SPL), Correlation Response-CR $(\%)$, Stimulus Level-STL (dB SPL) i Proportion of Stimulus and Noise-S/N (dB) at compensated ( $\mathrm{k}$ ) and non-compensated ( $\mathrm{n}$ ) pressure by groups of diseases (inflammatory-Z and non-inflammatory-NZ)

Table 2. Correlation of parameters TEOAE at non-compensated and compensated pressure

\begin{tabular}{|c|c|c|c|c|c|c|c|c|}
\hline $\mathbf{r}$ & CR & STL & $\mathbf{A}$ & $\mathbf{S} / \mathbf{N}$ & $\mathrm{S} / \mathrm{N} \_0.5$ & $\mathrm{~S} / \mathrm{N} \_1.5$ & $\mathrm{~S} / \mathrm{N} \_2.5$ & $\mathrm{~S} / \mathrm{N} \_3.5$ \\
\hline CR & - & $0.52 * * *$ & $0.32 * * *$ & $0.25 * *$ & $0.45 * * *$ & $0.13^{\text {ns }}$ & $0.44 * * *$ & $0.51 * * *$ \\
\hline STL & $0.52 * * *$ & - & $0.17 *$ & $0.13^{\mathrm{ns}}$ & $-0.02^{\mathrm{ns}}$ & $-0.03^{\text {ns }}$ & $0.17^{\mathrm{ns}}$ & $0.13^{*}$ \\
\hline $\mathbf{A}$ & $0.22 * *$ & $0.08^{\mathrm{ns}}$ & - & $0.38 * * *$ & $0.27 * * *$ & $0.15^{\mathrm{ns}}$ & $0.31 * * *$ & $0.26^{* *}$ \\
\hline $\mathbf{S} / \mathbf{N}$ & $0.13 *$ & $-0.05^{\text {ns }}$ & $0.17 *$ & - & $0.25 * *$ & $0.16^{*}$ & $0.27 * * *$ & $0.28 * * *$ \\
\hline S/N_0.5 & $0.46^{* * *}$ & $0.12^{\mathrm{ns}}$ & $0.14^{\mathrm{ns}}$ & $0.25 * *$ & - & $0.41 * * *$ & $0.53 * * *$ & $0.57 * * *$ \\
\hline $\mathrm{S} / \mathrm{N} \_1.5$ & $0.48 * * *$ & $0.15^{\mathrm{ns}}$ & $0.11^{\mathrm{ns}}$ & $0.19 *$ & $0.67 * * *$ & - & $0.56 * * *$ & $0.48 * * *$ \\
\hline $\mathrm{S} / \mathrm{N} \_2.5$ & $0.41 * * *$ & $0.04^{\mathrm{ns}}$ & $0.07^{\mathrm{ns}}$ & $0.18^{*}$ & $0.53 * * *$ & $0.68 * * *$ & - & $0.68 * * *$ \\
\hline
\end{tabular}




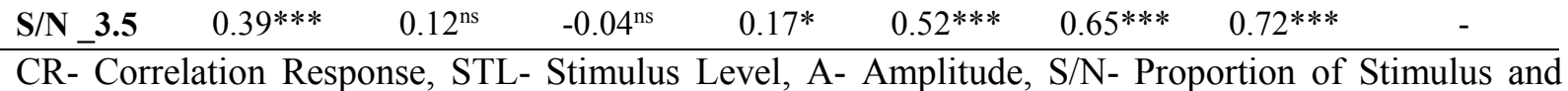
Noise, S/N ( $0.5, \ldots 1.5,2.5,3.5)$ - Proportion of Stimulus and Noise by different frequency; Statistical significance: ns- not significant, $*_{-} \mathrm{p}<0.05, * *_{-} \mathrm{p}<0.01, * * *_{-} \mathrm{p}<0.001$; The value of TEOAE above a diagonale with the compensated pressure, and value of TEOAE below a diagonale with non-compensated pressure.

Within both groups of diseases of ear conduction apparatus differences in amplitudes $(\mathrm{p}=0.0812$ and $\mathrm{p}=1.485)$ did not exist. Pressure compensation caused a statistically significantly higher value of the relationship of stimulus and noise $(\mathrm{S} / \mathrm{N})$ only in the group of non-inflammatory disorders $(\mathrm{p}=0.0312)$.

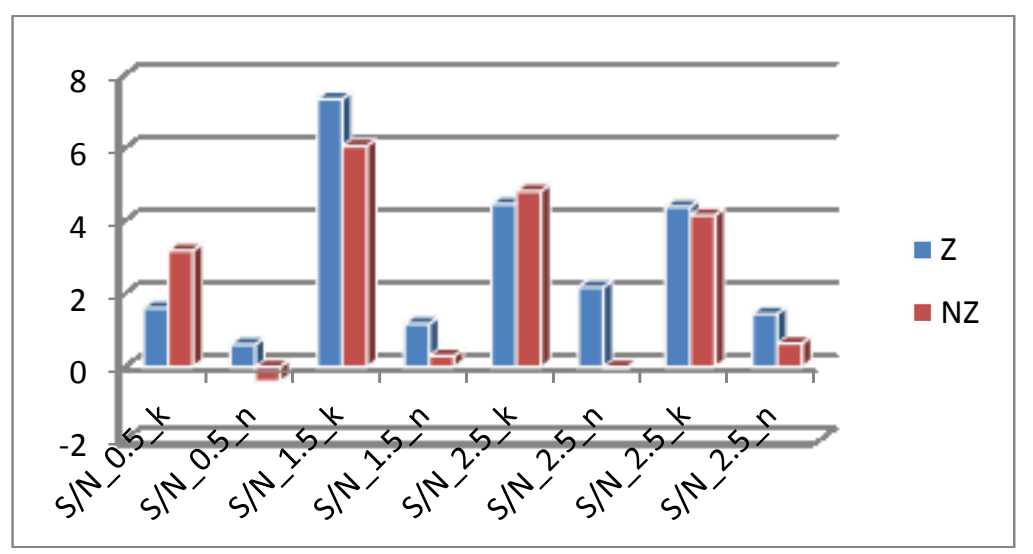

Figure 4. Proportion of Stimulus and Noise $(\mathrm{S} / \mathrm{N}, \mathrm{dB})$ by different frequency $\left({ }_{0} 0.5, \ldots 1.5\right.$, $\left.2.5 \mathrm{i} \_3.5 \mathrm{kHz}\right)$ at compensated $(\mathrm{k})$ and non-compensated $(\mathrm{n})$ pressure by groups of diseases (inflammatory-Z and non-inflammatory-NZ)

Mean relationship of stimulus and noise at different frequencies at pressure compensation was higher in relation to the treatment at ambiental pressure (Figure 4). Within inflammatory disorders in middle ear the differences existed in $\mathrm{S} / \mathrm{N}$ values at frequencies of 1.5, 2.5 and $3.5 \mathrm{kHz}(\mathrm{p}<0.0001, \mathrm{p}=0.0247$ and $\mathrm{p}=0.0010)$. Regarding non-inflammatory processes, relationship of stimulus and noise at all frequencies was higher $(p=0.0003$, $\mathrm{p}=0.0019, \mathrm{p}=0.0002$ and $\mathrm{p}=0.0002$ ) during pressure compensation for $3.59,5.76,4.84$ and $4.52 \mathrm{~dB}$, respectively. 


\section{DISCUSSION}

The effect of pressure compensation has caused higher values of all registered TEOAE parameters in relation to emission parameters at ambiental pressure what is in concordance with various studies. Thus research (8) determined that pressure compensation in middle ear can increase TEOAE level at frequencies of 1,2 and $3 \mathrm{kHz}$ in $80 \%$ cases, while at the frequency of $4 \mathrm{kHz}$ the increase was observed in $58 \%$ and decrease in $42 \%$ cases.

Values of amplitude confirmed by our study are similar to the study of (9) in which TEOAE measuring in newborn babies showed general value amplitudes of about $30 \mathrm{~dB}$ SPL, while in adults the values from 8 to $12 \mathrm{~dB}$ SPL were determined. The increase in the amplitude at pressure compensation in middle ear in our study was $4.11 \mathrm{~dB}$ SPL, what is higher compared to study of (8) who established increase of 1.9 dB SPL at treatment with pressure compensation.

The results showed that between the treatments with pressure compensation there existed no significant differences in amplitudes so that obtained results partly correspond with the study of (8) where the highest increase was observed in the frequency range of 1 to 2 $\mathrm{kHz}$, a statistically significant correlation was perceived between compensated pressure and increased TEOAE amplitude. At higher frequencies (3 and $4 \mathrm{kHz}$ ), TEOAE amplitudes were not sensitive to pressure compensation. The study of (10) in which the patients with tympanometrical peak ranged from -100 to $-300 \mathrm{daPa}$ were tested, in all cases pressure compensation in middle ear increased the emission amplitudes. The study of (11) indicates 
that low values of negative pressure in middle ear can significantly affect spectrum of stimulus and response. At lower frequencies the compensation can result in the increase of the TEOAE amplitude compared with the amplitudes at ambiental pressure.

Some studies show that if the quantity of secretion in cavum tympani is elevated the decrease in amplitude and reproducibility of TEOAE response is also higher. Thus research (12) suggest that mucoid content in middle ear can reduce TEOAE more than non-mucoid secretion, while both can disable a proper transfer of sound wave to cochlear, and therefore its adequate treatment. Study by (13) indicates the differences in TEOAE registration between lesions such as tympanosclerosis, OMA, otosclerosis and chain discontinuity depending on the gravity of disease and the model utilised to register otoacoustic emissions. In our study a statistically significant differences in registered TEOAE parameters compared between the groups of non-inflammatory and inflammatory diseases within both treatments with pressure were not determined. It is possible that differences between individual lesions could be confirmed but in our study they were grouped due to a small sample of persons tested for singular diseases.

Low values of negative pressure in middle ear can spread to the TEOAE spectrum and potentially affect test accuracy. Study of (11) suggests that in longer monitoring (monitoring TEOAE) the pressure in middle ear should be close to ambiental pressure or compensated by equivalent pressure in external auditory canal.

\section{CONCLUSION}

Pressure compensation in middle ear has significantly affected obtaining TEOAE response and the size of this response. Mean values of correlation response and level of stimulus were significantly higher at pressure compensation regardless the disorder in ear conduction 
apparatus. In non-inflammatory diseases, increase in the relationship of stimulus and noise was significantly higher at all examined frequencies and in inflammatory processes at frequencies of $1.5,2.5$ and $3.5 \mathrm{kHz}$. Pressure compensation in middle ear caused higher values of all TEOAE parameters in reference to emission parameters at ambiental pressure.

\section{REFERENCES}

1. Kemp DT. Stimulated acoustic emissions from within the human auditory system. J Acoust Soc Am 1978; 64: 1386-91.

2. Silva GC, Delecrode RC, Kemp AT, Martins F, Cardoso ACV. Transient Evoked and Distortion Product Otoacoustic Emissions in a Group of Nenonates. Int Arch Otorhinolaryngol 2015; 19: 255-8. 
3. Mikić B, Đoković S, Sovilj M, Pantelić S. Otoakustička emisija kod neonatusa, dece i odraslih: In Otoakustička emisija, teorija i praksa (Ed. Jovičić S. and Sovilj M). Belgrade, IEFPG 2005; pp 122-42.

4. Jovičić S, Šarić Z, Subotić M. Metode merenja i obrade signala OAE: In Otoakustička emisija, teorija i praksa (Ed. Jovičić S. and Sovilj M). Belgrade, IEFPG 2005; pp 91121.

5. Glattke TJ, Kujawa SG. Otoacoustic emissions. Am J Audiol 1991; 1: 29-40.

6. Ho V, Daly KA, Hunter LL, Davey C. Otoacoustic emissions and tympanometry screening among 0-5 year olds. Laryngoscope 2002; 112: 513-9.

7. SAS Institute Inc (2002-2010). The SAS System for Windows, Cary, NC.

8. Hof JR, Anteunis LJ, Chenault MN, van Dijk P. Otoacoustic emissions at compensated middle ear pressure in children. Int J Audiol 2005; 44: 317-20.

9. Kok MR, van Zanten GA, Brocaar MP, Wallenburg HCS. Click evoked otoacoustic emissions in 1036 ears of healthy newborns. Audiology 1993; 32: 213-24.

10. Trine M B, Hirsh JE, Margolis RH. Effect of middle ear pressure on evoked otoacoustic emissions. Ear Hear 1993; 14: 401-7.

11. Marshall L, Heller LM , Westhusin LJ. Effect of negative middle-ear pressure on transient-evoked otoacoustic emissions. Ear Hear 1997; 18: 218-26.

12. Koivunen P, Uhari M, Laitakari K, Alho OP , Luotonen J. Otoacoustic emissions and tympanometry in children with otitis media. Ear Hear 2000; 21: 212-7.

13. Zhao F, Wada H, Koike T, Ohyama K. Transient evoked otoacoustic emissions in patients with middle ear disorders. Int J Audiol 2003; 42: 117-31. 
TEOAE at Non-compensated and Compensated Pressure 